\title{
Existence and Uniqueness of Almost Periodic Solutions for Neural Networks with Neutral Delays
}

\author{
Min Xu, Zengji Du, and Kaige Zhuang \\ School of Mathematics and Statistics, Jiangsu Normal University Xuzhou, Jiangsu 221116, China \\ Correspondence should be addressed to Zengji Du; duzengji@163.com
}

Received 13 December 2013; Accepted 18 February 2014; Published 17 April 2014

Academic Editor: Jifeng Chu

Copyright (C) 2014 Min Xu et al. This is an open access article distributed under the Creative Commons Attribution License, which permits unrestricted use, distribution, and reproduction in any medium, provided the original work is properly cited.

A class of neural networks system with neutral delays is investigated. The existence and uniqueness of almost periodic solution for the system are obtained by using fixed point theorem; we extend some results in the references.

\section{Introduction}

In recent years, neural networks have been deeply investigated due to their applicability in solving some image processing, signal processing, and pattern recognition problems. And neural networks have been applied in artificial intelligence and automatic control engineering because of their good abilities of information memory and information association $([1,2])$.

Cellular neural networks (for short CNN) have been introduced by Chua and Yang [3] in 1988. Usually, in the electronic implementations of analog neural networks, time delays will inevitably occur in the communication and response of neurons because of the unavoidable finite switching speed of amplifiers ([4-9]). Due to the complicated dynamic properties of the neural cells in the real world, some complicated dynamic properties have been described by delayed cellular neural networks (DCNNs) ([10-13]).

Bai [10] proposed a neural networks model which takes the following form:

$$
\begin{aligned}
x_{i}^{\prime}(t)= & -c_{i}(t) x_{i}(t)+\sum_{j=1}^{n} a_{i j}(t) f_{j}\left(x_{j}\left(t-\tau_{i j}(t)\right)\right) \\
& +\sum_{j=1}^{n} b_{i j}(t) g_{j}\left(x_{j}^{\prime}\left(t-\sigma_{i j}(t)\right)\right)+I_{i}(t),
\end{aligned}
$$

where $i=1,2, \ldots, n$, with initial condition

$$
\begin{gathered}
x_{i}(s)=\varphi_{i}(s), \quad s \in[-\tau, 0], \\
\varphi=\left(\varphi_{1}, \varphi_{2}, \ldots, \varphi_{n}\right)^{T} \in C^{1}\left([-\tau, 0], R^{n}\right) .
\end{gathered}
$$

By using fixed point theorem, Bai studied the global stability of almost periodic solutions for the above neural networks.

Since neural networks with neutral delays contain some very important information about the derivative of the past state, it is very important for us to study such complicated system. Some authors studied some more complicated neutral neural networks and several important results have been obtained in ([14-20]). For example, Pinto and Robledo [14] studied an impulsive neural network of $n$-units and distributed delays as follows:

$$
\begin{aligned}
x_{i}^{\prime}(t)= & -a_{i}(t) x_{i}(t) \\
& +\sum_{j=1}^{n}\left\{b_{i j}(t) f_{j}\left(x_{j}(t)\right)+c_{i j}(t) g_{j}\left(\left(k_{i j} * x_{j}\right)(t)\right)\right\} \\
& +\gamma_{i}(t), \quad t \neq t_{k}, \\
\Delta x_{i}\left(\tau_{k}\right)= & x_{i}\left(\tau_{k}^{+}\right)-x_{i}\left(\tau_{k}\right) \\
= & \sum_{j=1}^{n} A_{i j}(k) x_{j}\left(\tau_{k}\right)+I_{j}\left(x\left(\tau_{k}\right)\right)+\mu_{i}(k), \quad t=t_{k},
\end{aligned}
$$


where $\left(k_{i j} * x_{j}\right)(t)=\int_{0}^{\infty} k_{i j}(r) x_{j}(t-r) d r, x_{i}\left(\tau_{k}^{+}\right)=$ $\lim _{\delta \rightarrow 0^{+}} x_{i}\left(\tau_{k}+\delta\right), i=1,2, \ldots, n, k=1,2, \ldots$. Ву using spectral radius theorem they obtained a result on the existence and stability of an almost periodic solution for the system (3). follows:

Feng et al. [17] considered delayed neural network as

$$
\begin{aligned}
u_{i}^{\prime}(t)= & -c_{i} u_{i}(t)+\sum_{j=1}^{n} w_{i j} g_{j}\left(u_{j}(t)\right)+\sum_{j=1}^{n} a_{i j} g_{j}\left(u_{j}(t-\tau)\right) \\
& +\sum_{j=1}^{n} b_{i j} \int_{-\infty}^{t} k_{j}(t-s) g_{j}\left(u_{j}(s)\right) d s \\
& +\sum_{j=1}^{n} d_{i j} u_{j}^{\prime}(t-\tau)+\varrho_{j}, \\
u_{i}(t) & =\phi_{i}(t), \quad-\infty<t \leq 0, \quad i=1,2, \ldots, n,
\end{aligned}
$$

and obtained the system (4) having a unique equilibrium point, which is globally asymptotically stable.

Wang and Zhu [19] were concerned with the following generalized neutral-type neural networks with delays:

$$
\begin{aligned}
\left(A_{i} x_{i}\right)^{\prime}(t)= & -a_{i}(t) x_{i}(t) \\
& +\sum_{j=1}^{n}\left[b_{i j}(t) f_{j}\left(x_{j}(t)\right)\right. \\
& \left.+d_{i j}(t) g_{j}\left(x_{j}\left(t-\tau_{i j}(t)\right)\right)\right]+I_{i}(t), \\
x_{i}(t)= & \phi_{i}(t), \quad t \in[-r, 0], \quad i=1,2, \ldots, n,
\end{aligned}
$$

where $A_{i}$ is a difference defined by $\left(A_{i} x_{i}\right)(t)=x_{i}(t)-$ $\sum_{j=1}^{n} c_{i j}(t) x_{i}\left(t-\delta_{i j}(t)\right)$. By using fixed point theorem, Lyapunov function method, and comparison theorem, the authors studied the existence, global asymptotic stability, and exponential stability of almost periodic solution for the system (5).

Motivated by the above papers, in this paper, we consider the neural networks with neutral delays

$$
\begin{aligned}
& x_{i}^{\prime}(t)=-a_{i}(t) x_{i}(t)+\sum_{j=1}^{n} b_{i j}(t) f_{j}\left(x_{j}(t)\right) \\
&+\sum_{j=1}^{n} c_{i j}(t) f_{j}\left(x_{j}\left(t-\tau_{i j}(t)\right)\right) \\
&+\sum_{j=1}^{n} d_{i j}(t) \int_{-\infty}^{t} k_{j}(t-s) f_{j}\left(x_{j}(s)\right) d s \\
&+\sum_{j=1}^{n} e_{i j}(t) g_{j}\left(x_{j}^{\prime}\left(t-\sigma_{i j}(t)\right)\right)+I_{i}(t), \\
& i=1,2, \ldots, n,
\end{aligned}
$$

with initial condition

$$
x_{i}(s)=\psi_{i}(s), \quad s \in[-\tau, 0], i=1,2, \ldots, n,
$$

where $\psi=\left(\psi_{1}, \psi_{2}, \ldots, \psi_{n}\right)^{T} \in C^{1}\left([-\tau, 0], R^{n}\right), \tau=$ $\max _{1 \leq i, j \leq n}\left\{\sup _{t \in R} \tau_{i j}(t), \sup _{t \in R} \sigma_{i j}(t)\right\}>0, a_{i} \geq 0, \tau_{i j} \geq 0$, $\sigma_{i j} \geq 0, b_{i j}, c_{i j}, d_{i j}, e_{i j}$, and $I_{i}$ are almost periodic functions, $i, j=1,2, \ldots, n$, with ecological meaning are as follows:

$x_{i}(t)$ : the potential (or voltage) of cell $i$ at time $t$;

$a_{i}(t)$ : represents the rate with which the $i$ th unit will reset its potentialto the resting state in isolation when disconnected from the networkand external inputs at time $t$;

$b_{i j}(t), c_{i j}(t), d_{i j}(t), e_{i j}(t)$ : represent some strengths of connectivity and neutraldelayed strengths of connectivity between cell $i$ and $j$ at time $t$;

$f_{j}, g_{j}, k_{j}$ : the activation functions and $k_{j}$ is a scalar integrable function defined in $[0,+\infty)$;

$I_{i}(t)$ : an external input on the $i$ th unit at time $t$;

$\tau_{i j}, \sigma_{i j}$ : correspond to the transmission delays of the $i$ th unit along the axon of the $j$ th unit at time $t$.

The aim of this paper is to obtain sufficient conditions for the existence and uniqueness of almost periodic solutions to system (6), by using fixed point theorem and differential inequality theory and the analysis technique.

The remaining part of this paper is organized as follows. In Section 2, we will state several definitions and lemmas which will be useful in proving the main results. In Section 3, by using fixed point theorem and differential inequality techniques, the existence of almost periodic solution for system (6) is obtained. In Section 4, globally exponential stability of almost periodic solution for system (6) is obtained; thus the uniqueness of almost periodic solution for system (6) is obtained.

\section{Preliminaries}

For the sake of convenience, we introduce the following notations:

$$
\begin{gathered}
a_{i}^{-}=\inf _{t \in R}\left|a_{i}(t)\right|, \quad a_{i}^{+}=\sup _{t \in R}\left|a_{i}(t)\right|, \\
b_{i j}^{+}=\sup _{t \in R}\left|b_{i j}(t)\right|, \\
c_{i j}^{+}=\sup _{t \in R}\left|c_{i j}(t)\right|, \quad d_{i j}^{+}=\sup _{t \in R}\left|d_{i j}(t)\right|, \\
e_{i j}^{+}=\sup _{t \in R}\left|e_{i j}(t)\right|, \\
I_{i}^{+}=\sup _{t \in R}\left|I_{i}(t)\right|, \\
H=\max \left\{\max _{1 \leq i \leq n}\left\{\frac{I_{i}^{+}}{a_{i}^{-}}\right\}, \max _{1 \leq i \leq n}\left\{I_{i}^{+}\left(1+\frac{a_{i}^{+}}{a_{i}^{-}}\right)\right\}\right\},
\end{gathered}
$$




$$
\begin{array}{r}
\phi_{0}(t)=\left(\int_{-\infty}^{t} e^{-\int_{s}^{t} a_{1}(u) d u} I_{1}(s) d s, \ldots,\right. \\
\left.\int_{-\infty}^{t} e^{-\int_{s}^{t} a_{n}(u) d u} I_{n}(s) d s\right)^{T} .
\end{array}
$$

For system (6), we introduce the following assumptions. $\left(H_{1}\right) f_{j}(0)=0, g_{j}(0)=0, f_{j}, g_{j}: R \rightarrow R$ are Lipschitz continuous with Lipschitz constants $L_{j}^{f}$ and $L_{j}^{g}$, respectively, $\left|f_{j}(u)-f_{j}(v)\right| \leq L_{j}^{f}|u-v|$ and $\left|g_{j}(u)-g_{j}(v)\right| \leq L_{j}^{g}|u-v|$, for all $u, v \in R$ :

$$
\begin{aligned}
\left(H_{2}\right) \theta=\max & \left\{\max _{1 \leq i \leq n}\left\{\frac{1}{a_{i}^{-}}, 1+\frac{a_{i}^{+}}{a_{i}^{-}}\right\}\right. \\
& \left.\times\left[\sum_{j=1}^{n}\left(b_{i j}^{+} L_{j}^{f}+c_{i j}^{+} L_{j}^{f}+d_{i j}^{+} L_{j}^{f}+e_{i j}^{+} L_{j}^{g}\right)\right]\right\}
\end{aligned}
$$

$<1$.

$\left(H_{3}\right) \int_{0}^{+\infty} k_{j}(s) d s=1$ and $k_{j}(t)$ is a decreasing function about $t$.

In this paper, we will denote $\|m\|_{x}=\max \left\{\|m\|_{0},\left\|m^{\prime}\right\|_{0}\right\}$, where $\|m\|_{0}=\max _{1 \leq i \leq n} \sup _{t \in R}\left|m_{i}(t)\right|,\|m\|_{1}=\max \{\|m\|$, $\left.\left\|m^{\prime}\right\|\right\}$, where $\|m\|=\max _{1 \leq i \leq n}\left|m_{i}(t)\right|,\|M\|=$ $\sup _{x \in R^{n},\|x\|=1}|M x|$, where $M$ is matrix. Define the space $X$ as $X=\left\{\phi \mid \phi=\left(\phi_{1}(t), \phi_{2}(t), \ldots, \phi_{n}(t)\right)^{T}\right.$, where $\phi_{i}: R \rightarrow R$ is continuously differentiable almost periodic function\}; then $X$ is a Banach space with the norm defined by

$$
\begin{aligned}
\|\phi\|_{x} & =\max \left\{\|\phi\|_{0},\left\|\phi^{\prime}\right\|_{0}\right\} \\
& =\max \left\{\max _{1 \leq i \leq n} \sup _{t \in R}\left|\phi_{i}(t)\right|, \max _{1 \leq i \leq n} \sup _{t \in R}\left|\phi_{i}^{\prime}(t)\right|\right\} .
\end{aligned}
$$

We introduce some useful definitions and lemmas, which are important to establish our results.

Definition 1 (see $[21,22]$ ). Let $f: R \rightarrow R^{n}$ be continuous in $t, f(t)$ is said to be almost periodic on $R$ if, for any $\varepsilon>0$, the set $T(f, \varepsilon)=\{\tau:|f(t+\tau)-f(t)|<\varepsilon$, for all $t \in R\}$ is relatively dense; that is, for $\forall \varepsilon>0$, it is possible to find a real number $l=l(\varepsilon)>0$, for any interval with length $l(\varepsilon)$, there exists a number $\tau=\tau(\varepsilon)$ in this interval such that $\|f(t+\tau)-f(t)\|<\varepsilon$, for $\forall t \in R$.

Definition 2 (see $[21,22]$ ). Let $x \in R^{n}$ and $Q(t)$ be a $n \times n$ continuous matrix defined on $R$. The linear system

$$
x^{\prime}(t)=Q(t) x(t)
$$

is said to admit an exponential dichotomy on $R^{n}$, if there exist positive constants $k, \alpha$, projection $P$ and the fundamental solution matrix $X(t)$ of (11) satisfying

$$
\begin{gathered}
\left\|X(t) P X^{-1}(s)\right\| \leq k e^{-\alpha(t-s)}, \quad \text { for } t \geq s, \\
\left\|X(t)(I-P) X^{-1}(s)\right\| \leq k e^{-\alpha(s-t)}, \quad \text { for } t \leq s .
\end{gathered}
$$

Definition 3 (see [10]). Let $x^{*}(t)=\left(x_{1}^{*}(t), x_{2}^{*}(t), \ldots, x_{n}^{*}(t)\right)^{T}$ be a continuously differentiable almost periodic solution of system (6) with initial value $\varphi^{*}=\left(\varphi_{1}^{*}(t), \varphi_{2}^{*}(t), \ldots, \varphi_{n}^{*}(t)\right)^{T} \epsilon$ $C^{1}\left([-\tau, 0] ; R^{n}\right)$. If there exist constants $\lambda>0$ and $M \geq 1$ such that for every solution $x(t)=\left(x_{1}(t), x_{2}(t), \ldots, x_{n}(t)\right)^{T}$ of system (6) with any initial value $\varphi=\left(\varphi_{1}(t), \varphi_{2}(t), \ldots, \varphi_{n}(t)\right)^{T} \in$ $C^{1}\left([-\tau, 0] ; R^{n}\right)$,

$$
\begin{aligned}
\left\|x(t)-x^{*}(t)\right\|_{1} & =\max \left\{\left\|x(t)-x^{*}(t)\right\|,\left\|x^{\prime}(t)-x^{* \prime}(t)\right\|\right\} \\
& \leq M\left\|\varphi-\varphi^{*}\right\|_{1} e^{-\lambda t}, \quad \forall t>0
\end{aligned}
$$

where $\left\|x(t)-x^{*}(t)\right\|=\max _{1 \leq i \leq n}\left|x_{i}(t)-x_{i}^{*}(t)\right|$. Then $x^{*}(t)$ is said to be globally exponential stable.

Lemma 4 (see $[21,22]$ ). If the linear system (11) admits an exponential dichotomy, then almost periodic system

$$
x^{\prime}(t)=Q(t) x(t)+g(t),
$$

has a unique almost periodic solution $x(t)$, and

$$
\begin{aligned}
x(t)= & \int_{-\infty}^{t} X(t) P X^{-1}(s) g(s) d s \\
& -\int_{t}^{+\infty} X(t)(I-P) X^{-1}(s) g(s) d s .
\end{aligned}
$$

Lemma 5 (see $[21,22]$ ). Let $c_{i}(t)$ be an almost periodic function on $R^{n}$ and

$$
M\left[c_{i}\right]=\lim _{T \rightarrow+\infty} \frac{1}{T} \int_{t}^{t+T} c_{i}(s) d s>0, \quad i=1,2, \ldots, n
$$

Then the linear system

$$
x^{\prime}(t)=\operatorname{diag}\left(-c_{1}(t),-c_{2}(t), \ldots,-c_{n}(t)\right) x(t)
$$

admits an exponential dichotomy on $R^{n}$.

\section{Existence of Almost Periodic Solution}

Theorem 6. Assume that $\left(H_{1}\right)-\left(H_{3}\right)$ hold; then there exists a unique continuously differentiable almost periodic solution of system (6) in the region $X_{0}=\left\{\phi \mid \phi \in X,\left\|\phi-\phi_{0}\right\|_{x} \leq \theta H /(1-\right.$ $\theta)\}$. 
Proof. For $\forall \phi \in X$, we consider the almost periodic solution $x^{\phi}(t)$ of nonlinear almost periodic differential equations

$$
\begin{aligned}
x_{i}^{\prime}(t)= & -a_{i} x_{i}(t)+\sum_{j=1}^{n} b_{i j} f_{j}\left(\phi_{j}(t)\right) \\
& +\sum_{j=1}^{n} c_{i j} f_{j}\left(\phi_{j}\left(t-\tau_{i j}(t)\right)\right) \\
& +\sum_{j=1}^{n} d_{i j} \int_{-\infty}^{t} k_{j}(t-s) f_{j}\left(\phi_{j}(s)\right) d s \\
& +\sum_{j=1}^{n} e_{i j} g_{j}\left(\phi_{j}^{\prime}\left(t-\sigma_{i j}\right)\right)+I_{i}(t),
\end{aligned}
$$

where $i=1,2, \ldots, n$.

From $a_{i}(t)>0$, we have

$$
M\left[a_{i}\right]=\lim _{T \rightarrow+\infty} \frac{1}{T} \int_{t}^{t+T} a_{i}(s) d s>0, \quad i=1,2, \ldots, n .
$$

From Lemmas 4 and 5, system (6) has a unique almost periodic solution $x^{\phi}(t)$ which can be expressed as follows:

$$
\begin{aligned}
& x^{\phi}(t) \\
& =\left(x_{1}^{\phi}(t), x_{2}^{\phi}(t), \ldots, x_{n}^{\phi}(t)\right)^{T} \\
& =\left(\int_{-\infty}^{t} e^{-\int_{s}^{t} a_{1}(u) d u}\right. \\
& \times\left[\sum_{j=1}^{n} b_{1 j} f_{j}\left(\phi_{j}(s)\right)\right. \\
& +\sum_{j=1}^{n} c_{1 j} f_{j}\left(\phi_{j}\left(s-\tau_{1 j}(s)\right)\right) \\
& +\sum_{j=1}^{n} d_{1 j} \int_{-\infty}^{s} k_{j}(s-u) f_{j}\left(\phi_{j}(u)\right) d u \\
& \left.+\sum_{j=1}^{n} e_{1 j} g_{j}\left(\phi_{j}^{\prime}\left(s-\sigma_{1 j}\right)\right)+I_{1}(s)\right] d s, \\
& \int_{-\infty}^{t} e^{-\int_{s}^{t} a_{2}(u) d u} \\
& \times\left[\sum_{j=1}^{n} b_{2 j} f_{j}\left(\phi_{j}(s)\right)\right. \\
& +\sum_{j=1}^{n} c_{2 j} f_{j}\left(\phi_{j}\left(s-\tau_{2 j}(s)\right)\right)
\end{aligned}
$$

$$
\begin{gathered}
+\sum_{j=1}^{n} d_{2 j} \int_{-\infty}^{s} k_{j}(s-u) f_{j}\left(\phi_{j}(u)\right) d u \\
\left.+\sum_{j=1}^{n} e_{2 j} g_{j}\left(\phi_{j}^{\prime}\left(s-\sigma_{2 j}\right)\right)+I_{2}(s)\right] d s, \ldots, \\
\int_{-\infty}^{t} e^{-\int_{s}^{t} a_{n}(u) d u} \\
\times\left[\sum_{j=1}^{n} b_{n j} f_{j}\left(\phi_{j}(s)\right)\right. \\
+\sum_{j=1}^{n} c_{n j} f_{j}\left(\phi_{j}\left(s-\tau_{n j}(s)\right)\right. \\
+\sum_{j=1}^{n} d_{n j} \int_{-\infty}^{s} k_{j}(s-u) f_{j}\left(\phi_{j}(u)\right) d u \\
+\sum_{j=1}^{n} e_{n j} g_{j}\left(\phi_{j}^{\prime}\left(s-\sigma_{n j}\right)\right) \\
\left.\left.\left.+I_{n}(s)\right)\right] d s\right) .
\end{gathered}
$$

Define an operator: $T: X \rightarrow X$ by setting

$$
(T \phi)(t)=x^{\phi}(t), \quad \forall \phi \in X
$$

By the definition of $\|\cdot\|_{x}$, one has

$$
\begin{aligned}
& \left\|\phi_{0}\right\|_{x} \\
& =\max \left\{\left\|\phi_{0}\right\|_{0},\left\|\phi_{0}^{\prime}\right\|_{0}\right\} \\
& =\max \left\{\max _{1 \leq i \leq n} \sup _{t \in R}\left\{\left|\int_{-\infty}^{t} I_{i}(s) e^{-\int_{s}^{t} a_{i}(u) d u} d s\right|\right\},\right. \\
& \max _{1 \leq i \leq n},\left\{\sup _{t \in R}\left\{\left|I_{i}(t)-\int_{-\infty}^{t} I_{i}(s) a_{i}(t) e^{-\int_{s}^{t} a_{i}(u) d u} d s\right|\right\}\right\} \\
& \leq \max \left\{\max _{1 \leq i \leq n}\left\{\frac{I_{i}^{+}}{a_{i}^{-}}\right\}, \max _{1 \leq i \leq n}\left\{I_{i}^{+}+a_{i}^{+} \frac{I_{i}^{+}}{a_{i}^{-}}\right\}\right\}=H .
\end{aligned}
$$

Hence, for $\forall \phi \in X_{0}=\left\{\phi \mid \phi \in X,\left\|\phi-\phi_{0}\right\|_{x} \leq \theta H /(1-\theta)\right\}$, one has

$$
\|\phi\|_{x} \leq\left\|\phi-\phi_{0}\right\|_{x}+\left\|\phi_{0}\right\|_{x} \leq \frac{\theta H}{1-\theta}+H=\frac{H}{1-\theta} .
$$

Now we prove that $T$ maps the set $X_{0}$ into itself. 
Obviously, for all $\phi \in X_{0}$, it follows from $\left(H_{1}\right)-\left(H_{3}\right)$ that

$$
\begin{aligned}
\left\|T \phi-\phi_{0}\right\|_{0} & \max _{1 \leq i \leq n, \operatorname{up}_{t \in R}}\left\{\mid \int_{-\infty}^{t} e^{-\int_{s}^{t} a_{i}(u) d u}\right. \\
\times & {\left[\sum_{j=1}^{n} b_{i j} f_{j}\left(\phi_{j}(s)\right)\right.} \\
& +\sum_{j=1}^{n} c_{i j} f_{j}\left(\phi_{j}\left(s-\tau_{i j}(s)\right)\right) \\
& +\sum_{j=1}^{n} d_{i j} \int_{-\infty}^{s} k_{j}(s-u) f_{j}\left(\phi_{j}(u)\right) d u \\
& \left.\left.+\sum_{j=1}^{n} e_{i j} g_{j}\left(\phi_{j}^{\prime}\left(s-\sigma_{i j}\right)\right)\right] d s \mid\right\}
\end{aligned}
$$$$
\leq \max _{1 \leq i \leq n} \sup _{t \in R}\left\{\int_{-\infty}^{t} e^{-\int_{s}^{t} a_{i}(u) d u}\right.
$$$$
\times\left[\sum_{j=1}^{n}\left|b_{i j}\right|\left|f_{j}\left(\phi_{j}(s)\right)\right|\right.
$$$$
+\sum_{j=1}^{n}\left|c_{i j}\right|\left|f_{j}\left(\phi_{j}\left(s-\tau_{i j}(s)\right)\right)\right|
$$$$
+\sum_{j=1}^{n}\left|d_{i j}\right|\left|f_{j}\left(\phi_{j}(s)\right)\right|
$$$$
\left.\left.+\sum_{j=1}^{n}\left|e_{i j}\right|\left|g_{j}\left(\phi_{j}^{\prime}\left(s-\sigma_{i j}\right)\right)\right|\right] d s\right\}
$$$$
\leq \max _{1 \leq i \leq n,} \sup _{t \in R}\left\{\int_{-\infty}^{t} e^{-\int_{s}^{t} a_{i}(u) d u}\right.
$$$$
\times\left[\sum_{j=1}^{n} b_{i j}^{+} L_{j}^{f}\|\phi\|_{0}\right.
$$$$
+\sum_{j=1}^{n} c_{i j}^{+} L_{j}^{f}\|\phi\|_{0}+\sum_{j=1}^{n} d_{i j}^{+} L_{j}^{f}\|\phi\|_{0}
$$$$
\left.\left.+\sum_{j=1}^{n} e_{i j}^{+} L_{j}^{g}\left\|\phi^{\prime}\right\|_{0}\right] d s\right\}
$$

$$
\begin{aligned}
\leq \max _{1 \leq i \leq n} \sup _{t \in R}\left\{\int_{-\infty}^{t} e^{-\int_{s}^{t} a_{i}(u) d u}\right. \\
\times\left[\sum_{j=1}^{n}\left(b_{i j}^{+} L_{j}^{f}+c_{i j}^{+} L_{j}^{f}+d_{i j}^{+} L_{j}^{f}+e_{i j}^{+} L_{j}^{g}\right)\right.
\end{aligned}
$$

$$
\begin{gathered}
\left.\left.\times\|\phi\|_{x}\right] d s\right\} \\
\leq \max _{1 \leq i \leq n} \sup _{t \in R}\left\{\int_{-\infty}^{t} e^{-(t-s) a_{i}^{-}} d s\right. \\
\left.\times \sum_{j=1}^{n}\left(b_{i j}^{+} L_{j}^{f}+c_{i j}^{+} L_{j}^{f}+d_{i j}^{+} L_{j}^{f}+e_{i j}^{+} L_{j}^{g}\right)\|\phi\|_{x}\right\} \\
=\max _{1 \leq i \leq n}\left\{\frac{1}{a_{i}^{-}} \sum_{j=1}^{n}\left(b_{i j}^{+} L_{j}^{f}+c_{i j}^{+} L_{j}^{f}+d_{i j}^{+} L_{j}^{f}+e_{i j}^{+} L_{j}^{g}\right)\right\}\|\phi\|_{x} .
\end{gathered}
$$

Moreover, we get

$$
\begin{gathered}
\left\|\left(T \phi-\phi_{0}\right)^{\prime}\right\|_{0} \operatorname{maxsup}_{1 \leq i \leq n, \sin _{t \in R}}\left\{\mid \sum_{j=1}^{n} b_{i j} f_{j}\left(\phi_{j}(t)\right)\right. \\
+\sum_{j=1}^{n} c_{i j} f_{j}\left(\phi_{j}\left(t-\tau_{i j}(t)\right)\right) \\
+\sum_{j=1}^{n} d_{i j} \int_{-\infty}^{t} k_{j}(t-s) f_{j}\left(\phi_{j}(s)\right) d s \\
+\sum_{j=1}^{n} e_{i j} g_{j}\left(\phi_{j}^{\prime}\left(t-\sigma_{i j}\right)\right) \\
-\int_{-\infty}^{t} a_{i}(t) e^{-\int_{s}^{t} a_{i}(u) d u} \\
+\sum_{j=1}^{n} d_{i j} \int_{-\infty}^{s} k_{j}(s-u) f_{j}\left(\phi_{j}(u)\right) d u \\
+\sum_{j=1}^{n} c_{j=1}^{n} e_{i j} f_{j}\left(\phi_{j}\left(\phi_{j}(s)\right)\right.
\end{gathered}
$$$$
\leq \max _{1 \leq i \leq n}\left\{\sum_{j=1}^{n} b_{i j}^{+} L_{j}^{f}\|\phi\|_{0}+\sum_{j=1}^{n} c_{i j}^{+} L_{j}^{f}\|\phi\|_{0}\right.
$$$$
+\sum_{j=1}^{n} d_{i j}^{+} L_{j}^{f}\|\phi\|_{0}+\sum_{j=1}^{n} e_{i j}^{+} L_{j}^{g}\left\|\phi^{\prime}\right\|_{0}
$$ 
6

Abstract and Applied Analysis

$$
\begin{gathered}
+a_{i}^{+} \int_{-\infty}^{t} e^{-\int_{s}^{t} a_{i}(u) d u} \\
\times\left[\sum_{j=1}^{n} b_{i j}^{+} L_{j}^{f}\|\phi\|_{0}+\sum_{j=1}^{n} e_{i j}^{+} L_{j}^{g}\left\|\phi^{\prime}\right\|_{0}\right. \\
\left.\left.+\sum_{j=1}^{n} c_{i j}^{+} L_{j}^{f}\|\phi\|_{0}+\sum_{j=1}^{n} d_{i j}^{+} L_{j}^{f}\|\phi\|_{0}\right] d s\right\} \\
\leq \max _{1 \leq i \leq n}\left\{\left(1+\frac{a_{i}^{+}}{a_{i}^{-}}\right)\right. \\
\left.\times \sum_{j=1}^{n}\left(b_{i j}^{+} L_{j}^{f}+c_{i j}^{+} L_{j}^{f}+d_{i j}^{+} L_{j}^{f}+e_{i j}^{+} L_{j}^{g}\right)\|\phi\|_{x}\right\} .
\end{gathered}
$$

Thus, by (23), (24), (25), and $\left(\mathrm{H}_{2}\right)$, one has

$$
\begin{aligned}
& \left\|T \phi-\phi_{0}\right\|_{x} \\
& =\max \left\{\left\|T \phi-\phi_{0}\right\|_{0},\left\|\left(T \phi-\phi_{0}\right)^{\prime}\right\|_{0}\right\} \\
& \leq \max _{1 \leq i \leq n}\left\{\left(\max \left\{\frac{1}{a_{i}^{-}}, 1+\frac{a_{i}^{+}}{a_{i}^{-}}\right\}\right)\right. \\
& \left.\times\left[\sum_{j=1}^{n}\left(b_{i j}^{+} L_{j}^{f}+c_{i j}^{+} L_{j}^{f}+d_{i j}^{+} L_{j}^{f}+e_{i j}^{+} L_{j}^{g}\right)\right]\right\}\|\phi\|_{x} \\
& =\theta\|\phi\|_{x} \leq \frac{\theta H}{1-\theta},
\end{aligned}
$$

which implies that $T \phi \in X_{0}$. So, the operator $T$ is a selfoperator from $X_{0}$ to $X_{0}$.

Next, we prove that $T$ is a contraction operator of the $X_{0}$. In fact, in view of $\left(H_{1}\right)-\left(H_{3}\right)$, for all $\phi, \varphi \in x_{0}$, we have

$\|T \phi-T \varphi\|_{0}$

$$
\begin{aligned}
=\operatorname{maxsup}_{1 \leq i \leq n_{,} \in R}\left\{\mid \int_{-\infty}^{t} e^{-\int_{s}^{t} a_{i}(u) d u}\right. \\
\times\left[\sum_{j=1}^{n} b_{i j}\left(f_{j}\left(\phi_{j}(s)\right)-f_{j}\left(\varphi_{j}(s)\right)\right)\right. \\
+\sum_{j=1}^{n} c_{i j}\left(f_{j}\left(\phi_{j}\left(s-\tau_{i j}(s)\right)\right)\right. \\
+\sum_{j=1}^{n} d_{i j} \int_{-\infty}^{s} k_{j}(s-u)
\end{aligned}
$$

$$
\begin{aligned}
& \times\left(f_{j}\left(\phi_{j}(u)\right)\right. \\
& \left.-f_{j}\left(\varphi_{j}(u)\right)\right) d u \\
& +\sum_{j=1}^{n} e_{i j}\left(g_{j}\left(\phi_{j}^{\prime}\left(s-\sigma_{i j}\right)\right)\right. \\
& \left.\left.\left.-g_{j}\left(\varphi_{j}^{\prime}\left(s-\sigma_{i j}\right)\right)\right)\right] d s \mid\right\} \\
& \times\left[\sum_{j=1}^{n}\left|b_{i j}\right|\left|f_{j}\left(\phi_{j}(s)\right)-f_{j}\left(\varphi_{j}(s)\right)\right|\right. \\
& +\sum_{j=1}^{n}\left|c_{i j}\right| \mid f_{j}\left(\phi_{j}\left(s-\tau_{i j}(s)\right)\right) \\
& -f_{j}\left(\varphi_{j}\left(s-\tau_{i j}(s)\right)\right) \mid \\
& +\sum_{j=1}^{n}\left|d_{i j}\right|\left|f_{j}\left(\phi_{j}(s)\right)-f_{j}\left(\varphi_{j}(s)\right)\right| \\
& +\sum_{j=1}^{n}\left|e_{i j}\right| \mid g_{j}\left(\phi_{j}^{\prime}\left(s-\sigma_{i j}\right)\right) \\
& \left.\left.-g_{j}\left(\varphi_{j}^{\prime}\left(s-\sigma_{i j}\right)\right) \mid\right] d s \mid\right\}
\end{aligned}
$$$$
\leq \operatorname{maxsup}_{1 \leq i \leq n, t \in R}\left\{\mid \int_{-\infty}^{t} e^{-\int_{s}^{t} a_{i}(u) d u}\right.
$$

$\leq \max _{1 \leq i \leq n} \sup _{t \in R}\left\{\int_{-\infty}^{t} e^{-\int_{s}^{t} a_{i}(u) d u}\right.$

$\times\left[\sum_{j=1}^{n} b_{i j}^{+} L_{j}^{f}\|\phi-\varphi\|_{0}\right.$

$+\sum_{j=1}^{n} c_{i j}^{+} L_{j}^{f}\|\phi-\varphi\|_{0}$

$+\sum_{j=1}^{n} d_{i j}^{+} L_{j}^{f}\|\phi-\varphi\|_{0}$

$\left.\left.+\sum_{j=1}^{n} e_{i j}^{+} L_{j}^{g}\left\|(\phi-\varphi)^{\prime}\right\|_{0}\right] d s\right\}$

$\leq \max _{1 \leq i \leq n, n_{t \in R}}\left\{\int_{-\infty}^{t} e^{-\int_{s}^{t} a_{i}(u) d u}\right.$

$\times\left[\sum_{j=1}^{n}\left(b_{i j}^{+} L_{j}^{f}+c_{i j}^{+} L_{j}^{f}+d_{i j}^{+} L_{j}^{f}+e_{i j}^{+} L_{j}^{g}\right)\right]$ 


$$
\begin{aligned}
& \left.\times\|\phi-\varphi\|_{x} d s\right\} \\
& \leq \max _{1 \leq i \leq n, \sup _{t \in R}}\left\{\int_{-\infty}^{t} e^{-(t-s) a_{i}^{-}} d s\right. \\
& \times \sum_{j=1}^{n}\left(b_{i j}^{+} L_{j}^{f}+c_{i j}^{+} L_{j}^{f}+d_{i j}^{+} L_{j}^{f}+e_{i j}^{+} L_{j}^{g}\right) \\
& \left.\times\|\phi-\varphi\|_{x}\right\} \\
& \leq \max _{1 \leq i \leq n}\left\{\frac{1}{a_{i}^{-}} \sum_{j=1}^{n}\left(b_{i j}^{+} L_{j}^{f}+c_{i j}^{+} L_{j}^{f}+d_{i j}^{+} L_{j}^{f}+e_{i j}^{+} L_{j}^{g}\right)\right\} \\
& \times\|\phi-\varphi\|_{x} \\
& \leq \theta\|\phi-\varphi\|_{x}, \\
& \left\|(T \phi-T \varphi)^{\prime}\right\|_{0} \\
& =\max _{1 \leq i \leq n,} \sup _{t \in R}\left\{\mid \sum_{j=1}^{n} b_{i j}\left(f_{j}\left(\phi_{j}(t)\right)-f_{j}\left(\varphi_{j}(t)\right)\right)\right. \\
& +\sum_{j=1}^{n} c_{i j}\left(f_{j}\left(\phi_{j}\left(t-\tau_{i j}(t)\right)\right)\right. \\
& \left.-f_{j}\left(\varphi_{j}\left(t-\tau_{i j}(t)\right)\right)\right) \\
& +\sum_{j=1}^{n} d_{i j} \int_{-\infty}^{t} k_{j}(t-s) \\
& \times\left(f_{j}\left(\phi_{j}(s)\right)-f_{j}\left(\varphi_{j}(s)\right)\right) d s \\
& +\sum_{j=1}^{n} e_{i j}\left(g_{j}\left(\phi_{j}^{\prime}\left(t-\sigma_{i j}\right)\right)\right. \\
& \left.-g_{j}\left(\varphi_{j}^{\prime}\left(t-\sigma_{i j}\right)\right)\right) \\
& -\int_{-\infty}^{t} a_{i}(t) e^{-\int_{s}^{t} a_{i}(u) d u} \\
& \times\left[\sum _ { j = 1 } ^ { n } b _ { i j } \left(f_{j}\left(\phi_{j}(s)\right)\right.\right. \\
& \left.-f_{j}\left(\varphi_{j}(s)\right)\right) \\
& +\sum_{j=1}^{n} c_{i j}\left(f_{j}\left(\phi_{j}\left(s-\tau_{i j}(s)\right)\right)\right. \\
& \left.-f_{j}\left(\varphi_{j}\left(s-\tau_{i j}(s)\right)\right)\right) \\
& +\sum_{j=1}^{n} d_{i j} \int_{-\infty}^{s} k_{j}(s-u) \\
& \times\left(f_{j}\left(\phi_{j}(u)\right)\right.
\end{aligned}
$$

$$
\left.-f_{j}\left(\varphi_{j}(u)\right)\right) d u
$$$$
+\sum_{j=1}^{n} e_{i j}\left(g_{j}\left(\phi_{j}^{\prime}\left(s-\sigma_{i j}\right)\right)\right.
$$$$
\left.\left.\left.-g_{j}\left(\varphi_{j}^{\prime}\left(s-\sigma_{i j}\right)\right)\right)\right] d s \mid\right\}
$$$$
\leq \max _{1 \leq i \leq n} \sum_{j=1}^{n}\left(b_{i j}^{+} L_{j}^{f}+c_{i j}^{+} L_{j}^{f}+d_{i j}^{+} L_{j}^{f}+e_{i j}^{+} L_{j}^{g}\right)
$$$$
\times\|\phi-\varphi\|_{x}
$$$$
+\max _{1 \leq i \leq n} \sup _{t \in R}\left\{\int_{-\infty}^{t} a_{i}^{+} e^{-\int_{s}^{t} a_{i}(u) d u}\right.
$$$$
\times\left[\sum _ { j = 1 } ^ { n } \left(b_{i j}^{+} L_{j}^{f}+c_{i j}^{+} L_{j}^{f}\right.\right.
$$$$
\left.\left.+d_{i j}^{+} L_{j}^{f}+e_{i j}^{+} L_{j}^{g}\right)\right] d s
$$$$
\left.\times\|\phi-\varphi\|_{x}\right\}
$$$$
\leq \max _{1 \leq i \leq n}\left\{\left(1+\frac{a_{i}^{+}}{a_{i}^{-}}\right)\right.
$$$$
\times \sum_{j=1}^{n}\left(b_{i j}^{+} L_{j}^{f}+c_{i j}^{+} L_{j}^{f}\right.
$$$$
\left.\left.+d_{i j}^{+} L_{j}^{f}+e_{i j}^{+} L_{j}^{g}\right)\right\}
$$

$$
\begin{aligned}
& \times\|\phi-\varphi\|_{x} \\
\leq & \theta\|\phi-\varphi\|_{x} .
\end{aligned}
$$

Thus,

$$
\|T \phi-T \varphi\|_{x} \leq \theta\|\phi-\varphi\|_{x}
$$

In view of $\left(H_{2}\right)$, we have $\theta<1$; it means that the $T$ is a contraction operator. By Banach fixed point theorem, there exists a fixed point $z \in X_{0}$ such that $T z=z$, which implies system (6) has an almost periodic solution.

\section{Uniqueness of Almost Periodic Solution}

Theorem 7. Assume that $\left(H_{1}\right)-\left(H_{3}\right)$ hold; then system (6) has a unique continuously differentiable almost periodic solution $z(t)$ which is globally exponentially stable. 
Proof. It follows from Theorem 6 that system (6) has at least one almost periodic solution $z(t)=$ $\left(z_{1}(t), z_{2}(t), \ldots, z_{n}(t)\right)^{T} \in X_{0}$ with initial value $\mu(t)=$ $\left(\mu_{1}(t), \mu_{2}(t), \ldots, \mu_{n}(t)\right)^{T}$. Let $x(t)=\left(x_{1}(t), x_{2}(t), \ldots, x_{n}(t)\right)^{T}$ be an arbitrary solution of system (6) with initial value $\psi^{*}(t)=\left(\psi_{1}^{*}(t), \psi_{2}^{*}(t), \ldots, \psi_{n}^{*}(t)\right)^{T}$. Let $y_{i}(t)=x_{i}(t)-z_{i}(t)$, $\psi_{i}(t)=\psi_{i}^{*}(t)-\mu_{i}(t), i=1,2, \ldots, n$. Then

$$
\begin{aligned}
& y_{i}^{\prime}(s)+a_{i} y_{i}(s) \\
& =\sum_{j=1}^{n} b_{i j}\left[f_{j}\left(y_{j}(s)+z_{j}(s)\right)-f_{j}\left(z_{j}(s)\right)\right] \\
& +\sum_{j=1}^{n} c_{i j}\left[f_{j}\left(y_{j}\left(s-\tau_{i j}(s)\right)+z_{j}\left(s-\tau_{i j}(s)\right)\right)\right. \\
& \left.\quad-f_{j}\left(z_{j}\left(s-\tau_{i j}(s)\right)\right)\right] \\
& +\sum_{j=1}^{n} d_{i j} \int_{-\infty}^{s} k_{j}(s-u) \\
& \quad \times\left[f_{j}\left(y_{j}(u)+z_{j}(u)\right)-f_{j}\left(z_{j}(u)\right)\right] d u \\
& +\sum_{j=1}^{n} e_{i j}\left[g_{j}\left(y_{j}^{\prime}\left(s-\sigma_{i j}\right)+z_{j}^{\prime}\left(s-\sigma_{i j}\right)\right)\right. \\
& \left.\quad-g_{j}\left(z_{j}^{\prime}\left(s-\sigma_{i j}\right)\right)\right],
\end{aligned}
$$

$$
\begin{aligned}
G_{i}\left(\varepsilon_{i}\right)= & a_{i}^{-}-\varepsilon_{i}-\left(a_{i}^{+}+a_{i}^{-}\right) \\
& \times \sum_{j=1}^{n}\left(b_{i j}^{+} L_{j}^{f}+c_{i j}^{+} L_{j}^{f}+d_{i j}^{+} L_{j}^{f}+e_{i j}^{+} L_{j}^{g}\right) e^{\varepsilon_{i} \tau},
\end{aligned}
$$

where $\eta_{i}, \varepsilon_{i} \in[0, \infty), i=1,2, \ldots, n$. From $\left(H_{2}\right)$, we have

$$
\begin{array}{r}
F_{i}(0)=a_{i}^{-}-\sum_{j=1}^{n}\left(b_{i j}^{+} L_{j}^{f}+c_{i j}^{+} L_{j}^{f}+d_{i j}^{+} L_{j}^{f}+e_{i j}^{+} L_{j}^{g}\right)>0, \\
G_{i}(0)=a_{i}^{-}-\left(a_{i}^{+}+a_{i}^{-}\right) \\
\times \sum_{j=1}^{n}\left(b_{i j}^{+} L_{j}^{f}+c_{i j}^{+} L_{j}^{f}+d_{i j}^{+} L_{j}^{f}+e_{i j}^{+} L_{j}^{g}\right)>0, \\
i=1,2, \ldots, n .
\end{array}
$$

Since $F_{i}(\cdot)$ and $G_{i}(\cdot)$ are continuous on $[0, \infty)$ and $F_{i}\left(\eta_{i}\right)$, $G_{i}\left(\varepsilon_{i}\right) \rightarrow-\infty$ as $\eta_{i}, \varepsilon_{i} \rightarrow \infty$, there exist $\eta_{i}^{*}, \varepsilon_{i}^{*}>0$ such that $F_{i}\left(\eta_{i}^{*}\right)=G_{i}\left(\varepsilon_{i}^{*}\right)=0$ and $F_{i}\left(\eta_{i}\right)>0$ for $\eta_{i} \in\left(0, \eta_{i}^{*}\right)$, $G_{i}\left(\varepsilon_{i}\right)>0$ for $\varepsilon_{i} \in\left(0, \varepsilon_{i}^{*}\right)$. It is easy to check that $\xi=$ $\min \left\{\eta_{1}^{*}, \eta_{2}^{*}, \ldots, \eta_{n}^{*}, \varepsilon_{1}^{*}, \varepsilon_{2}^{*}, \ldots, \varepsilon_{n}^{*}\right\}$. We obtain

$$
F_{i}(\xi) \geq 0, \quad G_{i}(\xi) \geq 0, \quad i=1,2, \ldots, n .
$$

So, we can choose a positive constant $0<\lambda<$ $\min \left\{\xi, a_{1}^{-}, a_{2}^{-}, \ldots, a_{n}^{-}\right\}$such that $F_{i}(\lambda)>0, G_{i}(\lambda)>0$, which implies that

$$
\begin{gathered}
\frac{\sum_{j=1}^{n}\left(b_{i j}^{+} L_{j}^{f}+c_{i j}^{+} L_{j}^{f}+d_{i j}^{+} L_{j}^{f}+e_{i j}^{+} L_{j}^{g}\right) e^{\lambda \tau}}{a_{i}-\lambda}<1, \\
\left(1+\frac{a_{i}^{+}}{a_{i}^{-}-\lambda}\right) \sum_{j=1}^{n}\left(b_{i j}^{+} L_{j}^{f}+c_{i j}^{+} L_{j}^{f}+d_{i j}^{+} L_{j}^{f}+e_{i j}^{+} L_{j}^{g}\right) e^{\lambda \tau}<1 .
\end{gathered}
$$

By (29), we have

$$
\begin{aligned}
& y_{i}(t) \\
& =\psi_{i}(0) e^{-\int_{0}^{t} a_{i}(u) d u} \\
& +\int_{0}^{t} e^{-\int_{s}^{t} a_{i}(u) d u} \\
& \quad \times\left\{\sum_{j=1}^{n} b_{i j}\left[f_{j}\left(y_{j}(s)+z_{j}(s)\right)-f_{j}\left(z_{j}(s)\right)\right]\right. \\
& +\sum_{j=1}^{n} c_{i j}\left[f_{j}\left(y_{j}\left(s-\tau_{i j}(s)\right)+z_{j}\left(s-\tau_{i j}(s)\right)\right)\right. \\
& \left.\quad-f_{j}\left(z_{j}\left(s-\tau_{i j}(s)\right)\right)\right] \\
& \quad \sum_{j=1}^{n} d_{i j} \int_{-\infty}^{s} k_{j}(s-u) \\
& \quad \times\left[f_{j}\left(y_{j}(u)+z_{j}(u)\right)-f_{j}\left(z_{j}(u)\right)\right] d u \\
& +\sum_{j=1}^{n} e_{i j}\left[g_{j}\left(y_{j}^{\prime}\left(s-\sigma_{i j}\right)+z_{j}^{\prime}\left(s-\sigma_{i j}\right)\right)\right. \\
& \left.\left.-g_{j}\left(z_{j}^{\prime}\left(s-\sigma_{i j}\right)\right)\right]\right\} d s .
\end{aligned}
$$

Let $M=a_{i}^{-} / \sum_{j=1}^{n}\left(b_{i j}^{+} L_{j}^{f}+c_{i j}^{+} L_{j}^{f}+d_{i j}^{+} L_{j}^{f}+e_{i j}^{+} L_{j}^{g}\right)$.

By $\left(H_{2}\right)$ we have $M>1$ and

$$
\|y(t)\|_{1}=\|\psi\|_{1} \leq M\|\psi\|_{1} e^{-\lambda t}, \quad \forall t \in[-\tau, 0], \lambda>0 .
$$

We claim that

$$
\|y(t)\|_{1} \leq M\|\psi\|_{1} e^{-\lambda t}, \quad t>0 .
$$

To prove (36), we first prove for any $p>1$, the following inequality holds:

$$
\|y(t)\|_{1}<p M\|\psi\|_{1} e^{-\lambda t}, \quad t>0 .
$$


Otherwise, there must be some $t_{1}>0$ and some $i \epsilon$ $\{1,2, \ldots, n\}$, such that

$$
\begin{aligned}
\|y(t)\|_{1} & =\max \left\{\|y(t)\|,\left\|y^{\prime}(t)\right\|\right\} \\
& =\max \left\{\max _{1 \leq j \leq n}\left|y_{j}(t)\right|, \max _{1 \leq j \leq n}\left|y_{j}^{\prime}(t)\right|\right\} \\
& =\max \left\{\left|y_{i}\left(t_{1}\right)\right|,\left|y_{i}^{\prime}\left(t_{1}\right)\right|\right\} \\
& =p M\|\psi\|_{1} e^{-\lambda t_{1}} \\
& \leq p M\|\psi\|_{1} e^{-\lambda t}, \quad \forall t \in\left[-\tau, t_{1}\right] .
\end{aligned}
$$

By (33), (34), (38), $\left(H_{1}\right)$, and $\left(H_{3}\right)$, we have

$$
\begin{aligned}
& \left|y_{i}\left(t_{1}\right)\right| \\
& \leq\|\psi\|_{1} e^{-\int_{0}^{t_{1}} a_{i}(u) d u} \\
& +\int_{0}^{t_{1}} e^{-\int_{s}^{t_{1}} a_{i}(u) d u} \\
& \quad \times\left[\sum_{j=1}^{n} b_{i j}^{+} L_{j}^{f}\left|y_{j}(s)\right|\right. \\
& \quad+\sum_{j=1}^{n} c_{i j}^{+} L_{j}^{f}\left|y_{j}\left(s-\tau_{i j}(s)\right)\right| \\
& \quad+\sum_{j=1}^{n} d_{i j} \int_{-\infty}^{s} k_{j}(s-u) L_{j}^{f}\left|y_{j}(u)\right| d u \\
& \left.\quad+\sum_{j=1}^{n} e_{i j}^{+} L_{j}^{g}\left|y_{j}^{\prime}\left(s-\sigma_{i j}\right)\right|\right] d s
\end{aligned}
$$$$
\leq\|\psi\|_{1} e^{-a_{i} t_{1}}
$$$$
+\int_{0}^{t_{1}} e^{-\int_{s}^{t_{1}} a_{i}(u) d u}
$$$$
\times\left[\sum_{j=1}^{n} b_{i j}^{+} L_{j}^{f} p M\|\psi\|_{1} e^{-\lambda(s)}\right.
$$$$
+\sum_{j=1}^{n} c_{i j}^{+} L_{j}^{f} p M\|\psi\|_{1} e^{-\lambda\left(s-\tau_{i j}(s)\right)}
$$$$
+\sum_{j=1}^{n} d_{i j} \int_{-\infty}^{s} k_{j}(s-u) L_{j}^{f} p M\|\psi\|_{1} e^{-\lambda(u)} d u
$$$$
\left.+\sum_{j=1}^{n} e_{i j}^{+} L_{j}^{g} p M\|\psi\|_{1} e^{-\lambda\left(s-\sigma_{i j}\right)}\right] d s
$$

$$
\begin{gathered}
=p M\|\psi\|_{1} e^{-\lambda t_{1}} \\
\times\left\{\frac{1}{p M} e^{\left(\lambda-a_{i}\right) t_{1}}\right. \\
+\int_{0}^{t_{1}} e^{-\int_{s}^{t_{1}} a_{i}(u) d u} e^{\lambda\left(t_{1}-s\right)} \\
\times\left[\sum_{j=1}^{n} b_{i j}^{+} L_{j}^{f}+\sum_{j=1}^{n} c_{i j}^{+} L_{j}^{f} e^{\lambda \tau_{i j}}\right. \\
+\sum_{j=1}^{n} d_{i j} \int_{-\infty}^{s} k_{j}(s-u) L_{j}^{f} d u \\
\left.\left.+\sum_{j=1}^{n} e_{i j}^{+} L_{j}^{g} e^{\lambda \sigma_{i j}}\right] d s\right\}
\end{gathered}
$$$$
\leq p M\|\psi\|_{1} e^{-\lambda t_{1}}
$$$$
\times\left\{\frac{1}{M} e^{\left(\lambda-a_{i}\right) t_{1}}\right.
$$$$
+\int_{0}^{t_{1}} e^{-\left(t_{1}-s\right) a_{i}} e^{\lambda\left(t_{1}-s\right)}
$$$$
\times\left[\sum_{j=1}^{n} b_{i j}^{+} L_{j}^{f}+\sum_{j=1}^{n} c_{i j}^{+} L_{j}^{f} e^{\lambda \tau_{i j}}\right.
$$$$
+\sum_{j=1}^{n} d_{i j} \int_{-\infty}^{s} k_{j}(s-u) L_{j}^{f} d u
$$$$
\left.\left.+\sum_{j=1}^{n} e_{i j}^{+} L_{j}^{g} e^{\lambda \sigma_{i j}}\right] d s\right\}
$$

$\leq p M\|\psi\|_{1} e^{-\lambda t_{1}}$

$$
\begin{aligned}
& \times\left\{\frac{1}{M} e^{\left(\lambda-a_{i}\right) t_{1}}\right. \\
& +\frac{1}{a_{i}-1}\left(1-e^{\left(\lambda-a_{i}\right) t_{1}}\right) \\
& \times \sum_{j=1}^{n}\left(b_{i j}^{+} L_{j}^{f}+c_{i j}^{+} L_{j}^{f}\right. \\
& +d_{i j} \int_{-\infty}^{s} k_{j}(t-s) L_{j}^{f} d u \\
& \left.\left.+e_{i j}^{+} L_{j}^{g}\right) e^{\lambda \tau}\right\} \\
& \leq p M\|\psi\|_{1} e^{-\lambda t_{1}} \\
& \times\left\{\frac{1}{M} e^{\left(\lambda-a_{i}\right) t_{1}}\right.
\end{aligned}
$$


10

Abstract and Applied Analysis

$$
\left.\begin{array}{l}
+\frac{1}{a_{i}-1}\left(1-e^{\left(\lambda-a_{i}\right) t_{1}}\right) \\
\times \sum_{j=1}^{n}\left(b_{i j}^{+} L_{j}^{f}+c_{i j}^{+} L_{j}^{f}\right. \\
\left.\left.+d_{i j} L_{j}^{f}+e_{i j}^{+} L_{j}^{g}\right) e^{\lambda \tau}\right\} \\
\leq p M\|\psi\|_{1} e^{-\lambda t_{1}} \\
\times\left\{\left(\frac{1}{M}-\frac{\sum_{j=1}^{n}\left(b_{i j}^{+} L_{j}^{f}+c_{i j}^{+} L_{j}^{f}+d_{i j} L_{j}^{f}+e_{i j}^{+} L_{j}^{g}\right) e^{\lambda \tau}}{a_{i}-\lambda}\right)\right. \\
\quad \times e^{\left(\lambda-a_{i}\right) t_{1}} \\
\left.+\frac{\sum_{j=1}^{n}\left(b_{i j}^{+} L_{j}^{\mathrm{f}}+c_{i j}^{+} L_{j}^{f}+d_{i j} L_{j}^{f}+e_{i j}^{+} L_{j}^{g}\right) e^{\lambda \tau}}{a_{i}-\lambda}\right\} \\
\leq p M\|\psi\|_{1} e^{-\lambda t_{1}} \\
\times \frac{\sum_{j=1}^{n}\left(b_{i j}^{+} L_{j}^{f}+c_{i j}^{+} L_{j}^{f}+d_{i j} L_{j}^{f}+e_{i j}^{+} L_{j}^{g}\right) e^{\lambda \tau}}{a_{i}-\lambda}
\end{array}\right\}
$$

Direct differentiation of (34) gives

$$
\begin{aligned}
& y_{i}^{\prime}(t) \\
& =-a_{i}(t) \psi_{i}(0) e^{-\int_{0}^{t} a_{i}(u) d u} \\
& -\int_{0}^{t} a_{i}(t) e^{-\int_{s}^{t} a_{i}(u) d u} \\
& \times\left\{\begin{array}{l}
\sum_{j=1}^{n} b_{i j}\left[f_{j}\left(y_{j}(s)+z_{j}(s)\right)-f_{j}\left(z_{j}(s)\right)\right] \\
+\sum_{j=1}^{n} c_{i j}\left[f _ { j } \left(y_{j}\left(s-\tau_{i j}(s)\right)\right.\right. \\
+\sum_{j=1}^{n} e_{i j}\left[g_{j}\left(y_{j}^{\prime}\left(s-\sigma_{i j}\right)+z_{j}^{\prime}\left(s-\sigma_{i j}\right)\right)\right. \\
\left.\left.+\sum_{j=1}^{n} d_{i j} \int_{-\infty}^{s} k_{j}(s-u)\right)\right) \\
\times\left[f_{j}\left(y_{j}(u)+z_{j}(u)\right)-f_{j}\left(z_{j}(u)\right)\right] d u
\end{array}\right.
\end{aligned}
$$

$$
\begin{gathered}
\left.\left.-g_{j}\left(z_{j}^{\prime}\left(s-\sigma_{i j}\right)\right)\right]\right\} d s \\
+\sum_{j=1}^{n} b_{i j}\left[f_{j}\left(y_{j}(s)+z_{j}(s)\right)-f_{j}\left(z_{j}(s)\right)\right] \\
+\sum_{j=1}^{n} c_{i j}\left[f_{j}\left(y_{j}\left(s-\tau_{i j}(s)\right)+z_{j}\left(s-\tau_{i j}(s)\right)\right)\right. \\
\left.+f_{j}\left(z_{j}\left(s-\tau_{i j}(s)\right)\right)\right] \\
+\sum_{j=1}^{n} d_{i j} \int_{-\infty}^{s} k_{j}(s-u) \\
\times\left[f_{j}\left(y_{j}(u)+z_{j}(u)\right)-f_{j}\left(z_{j}(u)\right)\right] d u \\
+\sum_{j=1}^{n} e_{i j}\left[g _ { j } \left(y_{j}^{\prime}\left(s-\sigma_{i j}\right)\right.\right. \\
\left.\left.+z_{j}^{\prime}\left(s-\sigma_{i j}\right)\right)-g_{j}\left(z_{j}^{\prime}\left(s-\sigma_{i j}\right)\right)\right] .
\end{gathered}
$$

Thus, from (33), (40), and $\left(H_{1}\right)$, we obtain

$$
\begin{aligned}
& \left|y_{i}^{\prime}\left(t_{1}\right)\right| \\
& \leq a_{i}^{+}|| \psi \|_{1} e^{-\int_{0}^{t_{1}} a_{i}(u) d u} \\
& +\int_{0}^{t_{1}} a_{i}\left(t_{1}\right) e^{-\int_{s}^{t_{1}} a_{i}(u) d u} \\
& \quad \times\left[\sum_{j=1}^{n} b_{i j}^{+} L_{j}^{f}\left|y_{j}(s)\right|\right. \\
& +\sum_{j=1}^{n} c_{i j}^{+} L_{j}^{f}\left|y_{j}\left(s-\tau_{i j}(s)\right)\right| \\
& \left.+\sum_{j=1}^{n} d_{i j}\right]_{-\infty}^{s} k_{j}(s-u) L_{j}^{f}\left|y_{j}(u)\right| d u \\
& +\sum_{j=1}^{n} d_{i j} \int_{-\infty}^{t_{1}} e_{i j}^{+} L_{j}^{g}\left|y_{j}^{\prime}\left(t_{1}-\sigma_{i j}\right)\right| \\
& \left.+\sum_{j=1}^{n} c_{i j}^{+} e_{i j}^{+} L_{j}^{g}\left|y_{j}^{\prime}\left(s-\sigma_{i j}\right)\right|\right] d s \\
& +\sum_{j=1}^{n} b_{i j}^{+} L_{j}^{f}\left|y_{j}^{f}\left(t_{1}\right)\right| \\
& +y_{j}(u) \mid d u \\
& \left.+t_{1}-\tau_{i j}\left(t_{1}\right)\right) \mid
\end{aligned}
$$




$$
\begin{aligned}
& \leq a_{i}^{+}\|\psi\|_{1} e^{-a_{i}} t_{1} \\
& +\int_{0}^{t_{1}} a_{i}^{+} e^{-\int_{s}^{t_{1}} a_{i}(u) d u} \\
& \times\left[\sum_{j=1}^{n} b_{i j}^{+} L_{j}^{f} p M\|\psi\|_{1} e^{-\lambda(s)}\right. \\
& +\sum_{j=1}^{n} c_{i j}^{+} L_{j}^{f} p M\|\psi\|_{1} e^{-\lambda\left(s-\tau_{i j}(s)\right)} \\
& +\sum_{j=1}^{n} d_{i j} \int_{-\infty}^{s} k_{j}(s-u) L_{j}^{f} p M\|\psi\|_{1} e^{-\lambda(u)} d u \\
& \left.+\sum_{j=1}^{n} e_{i j}^{+} L_{j}^{g} p M\|\psi\|_{1} e^{-\lambda\left(s-\sigma_{i j}\right)}\right] d s \\
& +\sum_{j=1}^{n} b_{i j}^{+} L_{j}^{f} p M\|\psi\|_{1} e^{-\lambda\left(t_{1}\right)} \\
& +\sum_{j=1}^{n} c_{i j}^{+} L_{j}^{f} p M\|\psi\|_{1} e^{-\lambda\left(t_{1}-\tau_{i j}\left(t_{1}\right)\right)} \\
& +\sum_{j=1}^{n} d_{i j} \int_{-\infty}^{t_{1}} k_{j}\left(t_{1}-u\right) L_{j}^{f} p M\|\psi\|_{1} e^{-\lambda(u)} d u \\
& +\sum_{j=1}^{n} e_{i j}^{+} L_{j}^{g} p M\|\psi\|_{1} e^{-\lambda\left(t_{1}-\sigma_{i j}\right)} \\
& =p M\|\psi\|_{1} e^{-\lambda t_{1}} \\
& \times\left\{\frac{a_{i}^{+}}{p M} e^{\left(\lambda-a_{i}\right) t_{1}}\right. \\
& +\int_{0}^{t_{1}} a_{i}^{+} e^{-\int_{s}^{t_{1}} a_{i}(u) d u} e^{\lambda\left(t_{1}-s\right)} \\
& \times\left[\sum_{j=1}^{n} b_{i j}^{+} L_{j}^{f}+\sum_{j=1}^{n} c_{i j}^{+} L_{j}^{f} e^{\lambda \tau_{i j}}\right. \\
& +\sum_{j=1}^{n} d_{i j} \int_{-\infty}^{s} k_{j}(s-u) L_{j}^{f} d u \\
& \left.+\sum_{j=1}^{n} e_{i j}^{+} L_{j}^{g} e^{\lambda \sigma_{i j}}\right] d s \\
& +\sum_{j=1}^{n} b_{i j}^{+} L_{j}^{f}+\sum_{j=1}^{n} c_{i j}^{+} L_{j}^{f} e^{\lambda \tau_{i j}} \\
& +\sum_{j=1}^{n} d_{i j} \int_{-\infty}^{t_{1}} k_{j}\left(t_{1}-u\right) L_{j}^{f} d u \\
& \left.+\sum_{j=1}^{n} e_{i j}^{+} L_{j}^{g} e^{\lambda \sigma_{i j}}\right\}
\end{aligned}
$$

$$
\begin{aligned}
& \leq p M\|\psi\|_{1} e^{-\lambda t_{1}} \\
& \times\left\{\frac{a_{i}^{+}}{M} e^{\left(\lambda-a_{i}\right) t_{1}}\right. \\
& +\left(\sum_{j=1}^{n} b_{i j}^{+} L_{j}^{f}+\sum_{j=1}^{n} c_{i j}^{+} L_{j}^{f}\right. \\
& +\sum_{j=1}^{n} d_{i j} \int_{-\infty}^{s} k_{j}(s-u) L_{j}^{f} d u \\
& \left.+\sum_{j=1}^{n} e_{i j}^{+} L_{j}^{g}\right) e^{\lambda \tau} \\
& \times \int_{0}^{t_{1}} a_{i}^{+} e^{-\int_{s}^{t_{1}} a_{i}(u) d u} e^{\lambda\left(t_{1}-s\right)} d s \\
& +\sum_{j=1}^{n}\left(b_{i j}^{+} L_{j}^{f}+c_{i j}^{+} L_{j}^{f}\right. \\
& \left.\left.+d_{i j} \int_{-\infty}^{t_{1}} k_{j}\left(t_{1}-u\right) L_{j}^{f} d u+e_{i j}^{+} L_{j}^{g}\right) e^{\lambda \tau}\right\} \\
& =p M\|\psi\|_{1} e^{-\lambda t_{1}} \\
& \times\left\{\left(\frac{1}{M}-\frac{\sum_{j=1}^{n}\left(b_{i j}^{+} L_{j}^{f}+c_{i j}^{+} L_{j}^{f}+d_{i j} L_{j}^{f}+e_{i j}^{+} L_{j}^{g}\right) e^{\lambda \tau}}{a_{i}-\lambda}\right)\right. \\
& \times a_{i}^{+} e^{\left(\lambda-a_{i}\right) t_{1}} \\
& +\left(1+\frac{a_{i}^{+}}{a_{i}^{-}-\lambda}\right) \\
& \left.\times \sum_{j=1}^{n}\left(b_{i j}^{+} L_{j}^{f}+c_{i j}^{+} L_{j}^{f}+d_{i j} L_{j}^{f}+e_{i j}^{+} L_{j}^{g}\right) e^{\lambda \tau}\right\} \\
& <p M\|\psi\|_{1} e^{-\lambda t_{1}}\left(1+\frac{a_{i}^{+}}{a_{i}^{-}-\lambda}\right) \\
& \times \sum_{j=1}^{n}\left(b_{i j}^{+} L_{j}^{f}+c_{i j}^{+} L_{j}^{f}+d_{i j} L_{j}^{f}+e_{i j}^{+} L_{j}^{g}\right) e^{\lambda \tau} \\
& <p M\|\psi\|_{1} e^{-\lambda t_{1}}
\end{aligned}
$$

Therefore, in view of (39) and (41), we have

$$
\left\|y\left(t_{1}\right)\right\|_{1}=\max \left\{\left|y_{i}\left(t_{1}\right)\right|,\left|y_{i}^{\prime}\left(t_{1}\right)\right|\right\}<p M\|\psi\|_{1} e^{-\lambda t_{1}},
$$

which contradicts (38); that is, the inequality (37) holds. Letting $p \rightarrow 1$, then the inequality (36) holds. Hence, the almost periodic solution of system (6) is globally exponentially stable; that is, the almost periodic solution is unique.

Here we would like to give some remarks. 
Remark 8. If $b_{i j}=0$ and $d_{i j}=0$, the system (6) reduces to the system (1) in [10], and we improve the corresponding results of $[10]$.

Remark 9. When $g_{j}(x)=x$, the system (6) can be reduced to the system (3) in [17]. The methods and results in this paper are different from [17]. We use Banach fixed point theorem to study the existence and uniqueness of almost periodic solution for the system (6). Linear matrix inequalities and delaydependent conditions are given to guarantee the considered delayed neural network to have a unique equilibrium point, which is globally asymptotically stable in [17].

\section{An Example}

In this section, we give an example to illustrate the effectiveness of our results.

Example 10. Consider the neural networks with neutral delays:

$$
\begin{aligned}
x_{1}^{\prime}(t)= & -a_{1}(t) x_{1}(t)+\sum_{j=1}^{2} b_{1 j}(t) f_{j}\left(x_{j}(t)\right) \\
& +\sum_{j=1}^{2} c_{1 j}(t) f_{j}\left(x_{j}\left(t-\tau_{1 j}(t)\right)\right) \\
& +\sum_{j=1}^{2} d_{1 j} \int_{-\infty}^{t} k_{j}(t-s) f_{j}\left(x_{j}(s)\right) d s \\
& +\sum_{j=1}^{2} e_{1 j} g_{j}\left(x_{j}^{\prime}\left(t-\sigma_{1 j}(t)\right)\right)+I_{1}(t), \\
x_{2}^{\prime}(t)= & -a_{2}(t) x_{2}(t)+\sum_{j=1}^{2} b_{2 j}(t) f_{j}\left(x_{j}(t)\right) \\
& +\sum_{j=1}^{2} c_{2 j}(t) f_{j}\left(x_{j}\left(t-\tau_{2 j}(t)\right)\right) \\
& +\sum_{j=1}^{2} e_{2 j} g_{j}\left(x_{j}^{\prime}\left(t-\sigma_{2 j}(t)\right)\right)+I_{2}(t), k_{j}(t-s) f_{j}\left(x_{j}(s)\right) d s
\end{aligned}
$$

where

$$
\begin{gathered}
a_{1}(t)=\frac{2}{3}+\frac{1}{6} \cos t, \\
a_{2}(t)=\frac{5}{3}+\frac{5}{6} \cos t, \\
b_{11}=b_{12}=\frac{1}{2}-\frac{1}{6} \cos 3 t, \\
b_{21}=b_{22}=\frac{1}{2}-\frac{1}{5} \cos 3 t,
\end{gathered}
$$

$$
\begin{gathered}
c_{11}=c_{12}=\frac{1}{12} \sin ^{2} t, \\
c_{21}=c_{22}=\frac{1}{12} \cos ^{2} t, \\
d_{11}=d_{12}=\frac{1}{12} \cos ^{2}(\sqrt{7} t), \\
d_{21}=d_{22}=\frac{1}{12} \sin ^{2}(\sqrt{7} t), \\
e_{11}=e_{12}=\frac{1}{50} \sin |50 t|, \\
e_{21}=e_{22}=\frac{1}{50} \cos |50 t|, \\
I_{1}=\cos (2 t), \quad I_{1}=\sin (2 t), \\
k_{11}(t)=k_{12}(t)=k_{21}(t)=k_{22}(t)=e^{-t}, \\
f_{1}(x)=f_{2}(x)=\frac{1}{25}|x|, \\
\sigma_{21}=\tau_{22}=1+\frac{1}{2} \cos (\sqrt{2} t), \\
g_{1}(x)=g_{2}(x)=\frac{1}{25} \sin x, \\
\tau_{11}=1+\frac{1}{4} \sin (\sqrt{2} t), \\
\tau_{12}=1-\frac{1}{5} \cos \sqrt{8} t \\
\sigma_{22} \\
=1
\end{gathered}
$$

By simple calculation, we have

$$
\begin{aligned}
L_{1}=L_{2}=\frac{1}{25}, & l_{1}=l_{2}=\frac{1}{25}, \\
a_{1}^{-}=\frac{1}{2}, & a_{2}^{-}=\frac{5}{6}, \\
a_{1}^{+}=\frac{5}{6}, & a_{2}^{+}=\frac{5}{2}, \\
b_{11}^{+}=b_{12}^{+}=\frac{2}{3}, & b_{21}^{+}=b_{22}^{+}=\frac{7}{10}, \\
c_{11}^{+}=c_{12}^{+}=\frac{1}{12}, & c_{21}^{+}=c_{22}^{+}=\frac{1}{12}, \\
d_{11}^{+}=d_{12}^{+}=\frac{1}{12}, & d_{21}^{+}=d_{22}^{+}=\frac{1}{12}, \\
e_{11}^{+}=e_{12}^{+}=\frac{1}{50}, & e_{21}^{+}=e_{22}^{+}=\frac{1}{50}, \\
I_{1}^{+}=I_{2}^{+}=1, & \theta=0.284<1 .
\end{aligned}
$$

Clearly, $\left(H_{1}\right)-\left(H_{3}\right)$ hold. From Theorems 6 and 7, system (6) has a unique continuously differentiable almost periodic solution, which is globally exponentially stable. 


\section{Conclusion}

In this work, we are concerned with a neural network model with neutral delays. The existence and uniqueness of almost periodic solution for the system are explored by means of Banach fixed point theorem. Our result is in good agreement with some related results in the literature.

\section{Conflict of Interests}

The authors declare that there is no conflict of interests regarding the publication of this paper.

\section{Acknowledgments}

The authors express their sincere thanks to the anonymous reviewers for their valuable comments and suggestions for improving the quality of the paper. This work is supported by the Natural Science Foundation of china (Grant nos. 11071205 and 11101349) and partially supported by NSF of Jiangsu Province, PAPD of Jiangsu Higher Education Institutions, and Jiangsu Province postgraduate training project.

\section{References}

[1] B. Kosko, "Adaptive bidirectional associative memories," Applied Optics, vol. 26, no. 23, pp. 4947-4960, 1987.

[2] B. Kosko, "Bidirectional associative memories," IEEE Transactions on Systems, Man, and Cybernetics, vol. 18, no. 1, pp. 49-60, 1988.

[3] L. O. Chua and L. Yang, "Cellular neural networks: theory," IEEE Transactions on Circuits and Systems, vol. 35, no. 10, pp. 1257$1272,1988$.

[4] J. Cao, "Global asymptotic stability of delayed bi-directional associative memory neural networks," Applied Mathematics and Computation, vol. 142, no. 2-3, pp. 333-339, 2003.

[5] S. Arik, "Global asymptotic stability of hybrid bidirectional associative memory neural networks with time delays," Physics Letters A, vol. 351, no. 1-2, pp. 85-91, 2006.

[6] H. Yang, T. Chu, and C. Zhang, "Exponential stability of neural networks with variable delays via LMI approach," Chaos, Solitons and Fractals, vol. 30, no. 1, pp. 133-139, 2006.

[7] T. Liou, J. Yan, C. Cheng, and C. Huang, "Global exponential stability condition of a class of neuralnetworks with timevarying delays," Physics Letters A, vol. 399, no. 3-5, pp. 333-342, 2003.

[8] R. Sakthivel, A. Arunkumar, K. Mathiyalagan, and S. M. Anthoni, "Robust passivity analysis of fuzzy Cohen-Grossbert BAM neural networks with time-varying delays," Applied Mathematics and Computation, vol. 218, no. 7, pp. 3799-3809, 2011.

[9] K. Mathiyalagan, R. Sakthivel, and S. M. Anthoni, "New robust passivity criteria for stochastic fuzzy BAM neural networks with time-varying delays," Communications in Nonlinear Science and Numerical Simulation, vol. 17, no. 3, pp. 1392-1407, 2012.

[10] C. Bai, "Global stability of almost periodic solutions of Hopfield neural networks with neutral time-varying delays," Applied Mathematics and Computation, vol. 203, no. 1, pp. 72-79, 2008.

[11] B. Xiao, "Existence and uniqueness of almost periodic solutions for a class of Hopfield neural networks with neutral delays," Applied Mathematics Letters, vol. 22, no. 4, pp. 528-533, 2009.
[12] J. Qiu and J. Cao, "Delay-dependent robust stability of neutraltype neural networks with time delays," Journal of Mathematical Control Science and Applications, vol. 1, pp. 179-188, 2007.

[13] S. Xu, J. Lam, D. W. C. Ho, and Y. Zou, "Delay-dependent exponential stability for a class of neural networks with time delays," Journal of Computational and Applied Mathematics, vol. 183, no. 1, pp. 16-28, 2005.

[14] M. Pinto and G. Robledo, "Existence and stability of almost periodic solutions in impulsive neural network models," Applied Mathematics and Computation, vol. 217, no. 8, pp. 4167-4177, 2010.

[15] C. M. Marcus and R. M. Westervelt, "Stability of analog neural networks with delay," Physical Review A, vol. 39, no. 1, pp. 347359, 1989.

[16] Z. Zhang, K. Liu, and Y. Yang, "New LMI-based condition on global asymptotic stability concerning BAM neural networks of neutral type," Neurocomputing, vol. 81, pp. 24-32, 2012.

[17] J.-E. Feng, S. Xu, and Y. Zou, "Delay-dependent stability of neutral type neural networks with distributed delays," Neurocomputing, vol. 72, no. 10-12, pp. 2576-2580, 2009.

[18] J. Liu and G. Zong, "New delay-dependent asymptotic stability conditions concerning BAM neural networks of neutral type," Neurocomputing, vol. 72, no. 10-12, pp. 2549-2555, 2009.

[19] K. Wang and Y. Zhu, "Stability of almost periodic solution for a generalized neutral-type neural networks with delays," Neurocomputing, vol. 73, no. 16-18, pp. 3300-3307, 2010.

[20] K. Mathiyalagan, R. Sakthivel, and S. M. Anthoni, "Exponential stability result for discrete-time stochastic fuzzy uncertain neural networks," Physics Letters A, vol. 376, no. 8-9, pp. 901912, 2012

[21] A. M. Fink, Almost Periodic Differential Equations, vol. 377 of Lecture Notes in Mathematics, Springer, Berlin, Germany, 1974.

[22] C. Y. He, Almost Periodic Differential Equation, Higher Education Publishing House, Beijing, China, 1992 (Chinese). 


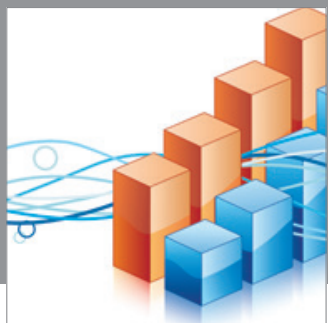

Advances in

Operations Research

mansans

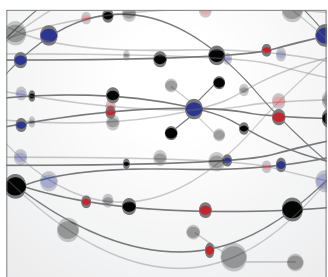

The Scientific World Journal
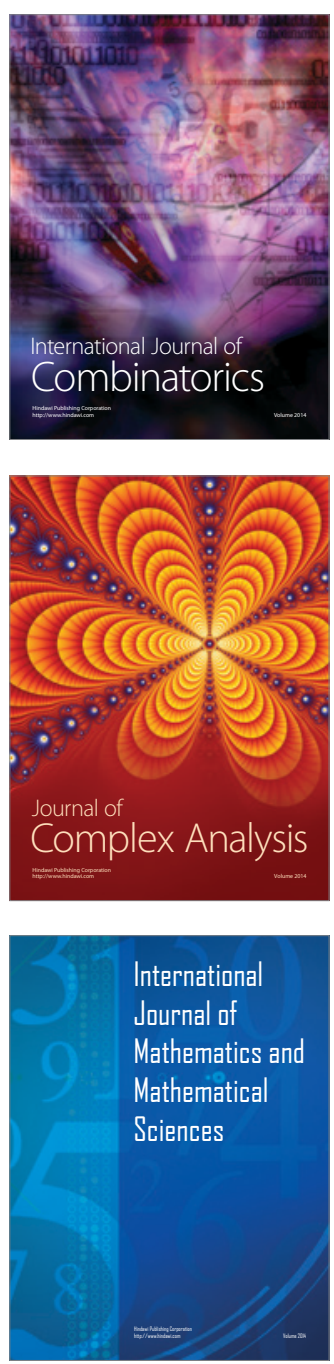
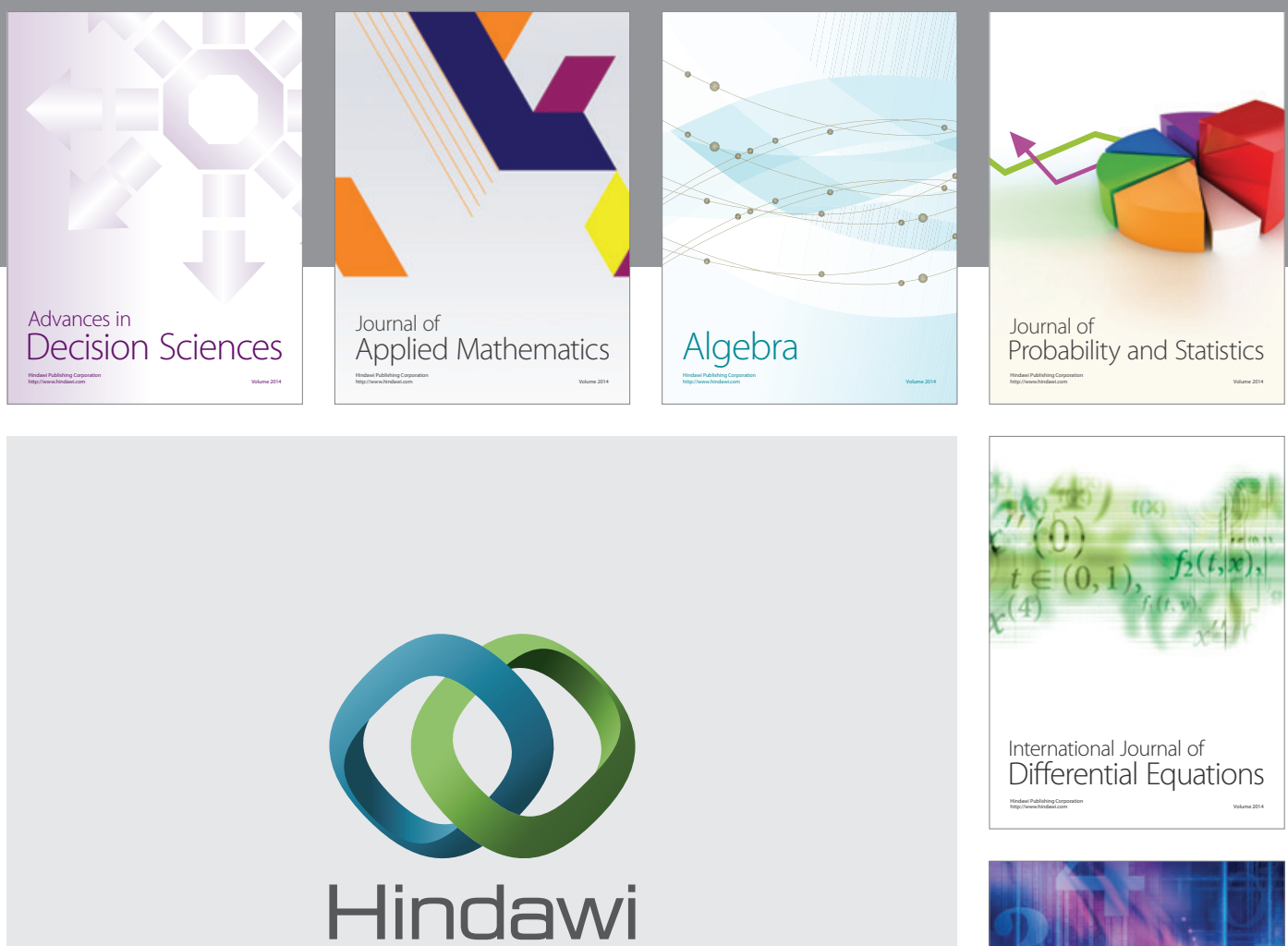

Submit your manuscripts at http://www.hindawi.com
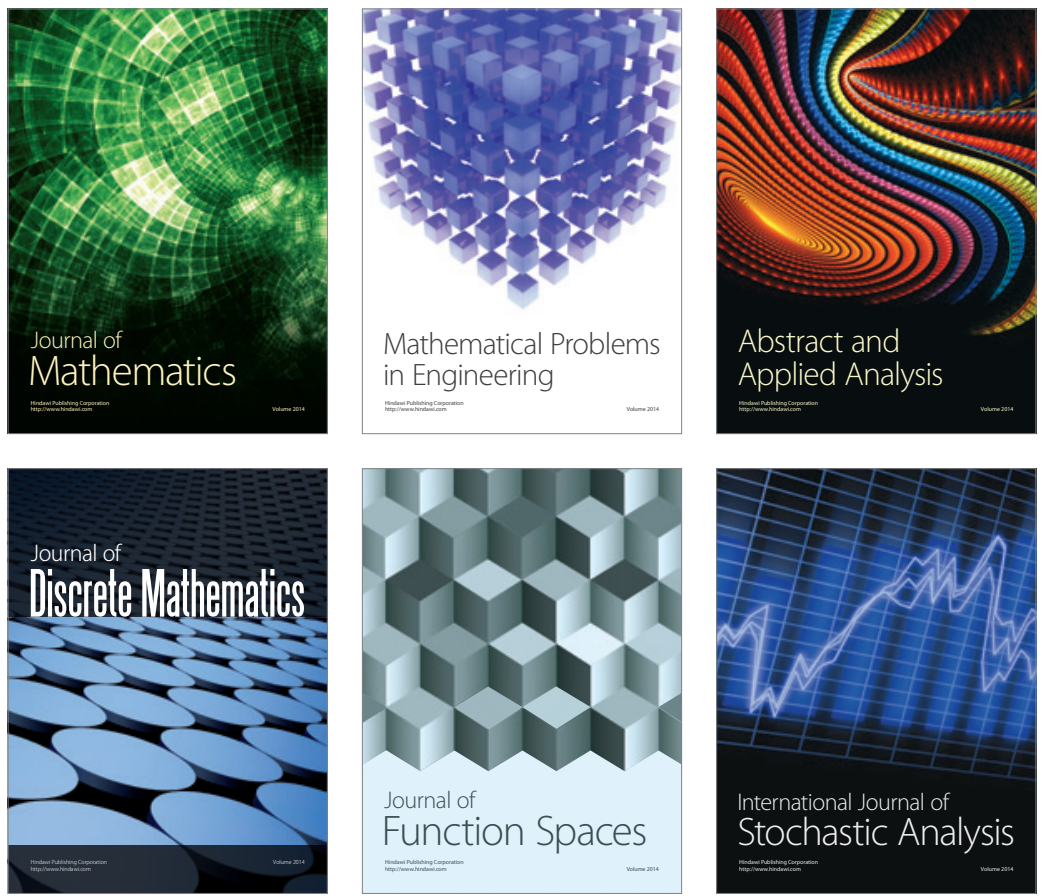

Journal of

Function Spaces

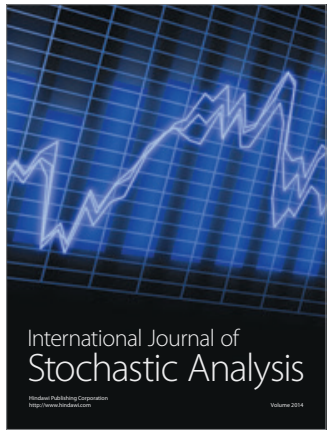

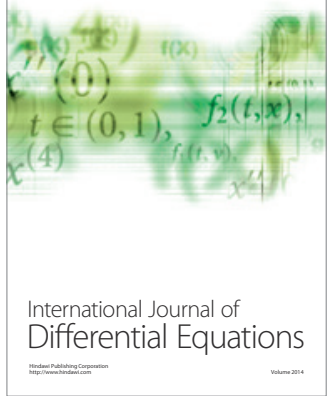
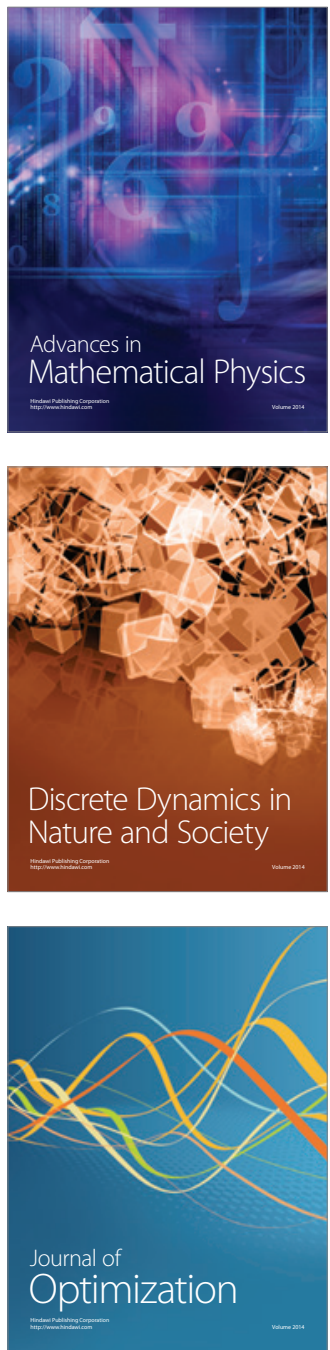